\title{
Uma Análise Inicial sobre a Aplicação de Blockchain na Sociedade
}

\author{
Emanuel F. Coutinho ${ }^{1}$, Wagner L. Braga Bezerra ${ }^{1}$, Delano Maia ${ }^{1}$ \\ ${ }^{1}$ Programa de Pós-Graduação em Computação (PCOMP) \\ Universidade Federal do Ceará (UFC) - Campus de Quixadá \\ emanuel.coutinhodufc.br, wagnerbragacrfealu.ufc.br, delanomaia@ufc.br
}

\begin{abstract}
By adding value to other emerging technologies, blockchain can impact many business sectors and society. Several areas are experiencing improvements in their processes and greater data security, such as health, education and finance. The objective of this work is to study the relationships and impacts of blockchain on society, mainly due to applications in the most diverse areas. For this, a literature research was performed, followed by the application of an online questionnaire on aspects of blockchain in society, and a qualitative analysis. As preliminary results, health and finance were the areas that most use blockchain, and the questionnaire revealed that it is important to study the impacts of blockchain on society.
\end{abstract}

Resumo. Ao agregar valor a outras tecnologias emergentes, a blockchain pode impactar muitos setores de negócios e a sociedade. Diversas áreas estão experimentando melhorias em seus processos e maior segurança dos dados, como saúde, educação e finanças. O objetivo deste trabalho é estudar as relações e impactos da blockchain sobre a sociedade, principalmente devido às aplicações nas mais diversas áreas. Para isso, uma pesquisa na literatura foi realizada, seguida da aplicação de um questionário online sobre aspectos da blockchain na sociedade, e uma análise qualitativa. Como resultados preliminares, saúde e finanças foram as áreas que mais utilizam blockchain, e o questionário revelou que é importante estudar os impactos da blockchain na sociedade.

\section{Introdução}

Com o amplo acesso provido pela internet, muitas vezes devido a um maior acesso a dispositivos móveis e serviços remotos, muitas pessoas passaram a conhecer mais sobre diversas tecnologias emergentes, como Computação em Nuvem, Internet das Coisas (IoT) e blockchain. Essas tecnologias podem ainda serem combinadas, gerando uma gama maior de serviços e produtos, impactando no cotidiano das pessoas.

Blockchain atualmente está sendo muito discutida tanto na academia quanto na indústria. E essa discussão está permeando todos os setores da sociedade. Um exemplo desse efeito, em algumas cidades, como Dubai, Estocolmo, Toronto e Visakhapatnam, propuseram projetos baseados em blockchain [Xie et al. 2019]. Em Dubai, o governo anunciou um plano para habilitar todas as transações e documentos governamentais na blockchain, tornando-a a primeira cidade digital movida a blockchain do mundo até 2020.

Blockchain é basicamente um banco de dados compartilhado imutável, descentralizado e público [Xie et al. 2019]. Blockchain possui alguns recursos que a tornam uma 
tecnologia atraente para enfrentar diversos desafios: (i) Descentralização: sistemas blockchain são executados em geral ponto a ponto sem um terceiro centralizado; (ii) Pseudonimato: na blockchain, cada nó é vinculado a um endereço público pseudônimo, mantendo sua identidade do mundo real oculta. Esse pseudonimato inerente é adequado para casos em que as identidades dos usuários devem ser mantidas em sigilo; (iii) Transparência: blockchain permite que todos acessem todos os registros de transação, o que a torna transparente. (iv) Democracia: algoritmos de consenso são executados por todos os nós descentralizados para chegar a um acordo antes que um bloco seja incluído na blockchain. Assim, as decisões são tomadas por todos os nós de maneira ponto a ponto, a tornando democratizada; (v) Segurança: em sistemas descentralizados baseados em blockchain é difícil ter um único ponto de falha. Assim, a segurança da rede é aprimorada; e (vi) Imutabilidade: na blockchain, todas as transações são assinadas usando assinaturas digitais. Além disso, os blocos de dados são vinculados e protegidos por meio de funções hash criptográficas unidirecionais. Qualquer modificação pequena gera um hash diferente e pode ser detectada imediatamente, tornando o livro razão compartilhado imutável.

Tais características estão possibilitando que diversas áreas tenham melhorias em seus processos e maior segurança na gestão dos dados. Áreas como finanças, saúde, educação e logística estão se apropriando da blockchain para o incremento de suas atividades e aplicações. Assim, a blockchain abriu uma série de possibilidades para empresas nas quais o valor pode ser transferido diretamente entre os participantes pela internet, da mesma maneira fácil como pagar em dinheiro e da mesma maneira conveniente que usar mensagens instantâneas sem intermediários ou pontos de controle centralizados [Aste et al. 2017]. Isso promove um novo modelo de negócio aos usuários.

No contexto da atual quarta revolução industrial, caracterizada pela fusão de diversas tecnologias que misturam o espaço físico e cibernético, a blockchain tem um papel mais amplo. Junto com outras tecnologias emergentes, como computação em névoa, aprendizado de máquina, inteligência artificial e veículos autônomos, a blockchain pode impactar muitos setores de negócios e a sociedade em geral [Aste et al. 2017].

Usualmente, nossa sociedade é centralizada, existindo hierarquias institucionais para governar atividades de nossas comunidades socioeconômicas [Aste et al. 2017]. Nesse contexto, a blockchain possibilita novos modelos de negócios, maneiras inovadoras de organizações, novos processos de trabalho e de produção nos quais o acesso e o compartilhamento é sobre propriedade. E assim, a blockchain cria a oportunidade para a geração de um nível de confiança necessário entre partes desconhecidas e anônimas, permitindo a negociação sem intermediários. Porém, os possíveis impactos da blockchain e suas aplicações na sociedade podem ser fundamentais e revolucionários, trazendo inevitavelmente desafios éticos imprevisíveis em um futuro previsível [Tang et al. 2019].

Nesse contexto, esse trabalho tem como objetivo apresentar um estudo sobre as relações e impactos da blockchain sobre a sociedade, seja por suas características, seja por aplicações nas mais diversas áreas. Para isso, uma pesquisa inicial na literatura será realizada, seguida da aplicação de um questionário online com algumas pessoas que já conhecem blockchain sobre aspectos da sociedade. Os resultados servirão para disseminar as possibilidades de aplicação da blockchain e provocar reflexões sobre seu uso, além de justificar a continuidade da pesquisa e seu aprofundamento. 
O restante deste trabalho está dividido nas seguintes seções: a Seção 2 discute alguns trabalhos relacionados; a Seção 3 descreve a metodologia aplicada a este trabalho; a Seção 4 apresenta os resultados; a Seção 5 relata algumas discussões e análises; e por fim, na Seção 6 as conclusões e trabalhos futuros são apresentados.

\section{Trabalhos Relacionados: Blockchain e Sociedade}

Esta seção descreve alguns trabalhos com ideias semelhantes a esta proposta, seja na justificativa da utilização da blockchain pela sociedade, seja pela relação de aplicações ou áreas de atuação onde blockchain pode ser utilizada. Os trabalhos foram obtidos a partir de buscas nas bases digitais IEEExplorer e ACM Digital Library, com a string de busca "blockchain AND society" e analisadas apenas pela leitura do conteúdo.

Blockchain abriu uma série de novas possibilidades para empresas nas quais o valor pode ser transferido diretamente entre os participantes pela internet [Aste et al. 2017]. Isso da mesma maneira que é fácil pagar em dinheiro ou conveniente como usar mensagens instantâneas, sem intermediários ou pontos de controle centralizados. Aplicativos blockchain baseados em organizações autônomas descentralizadas (DAOs) representam uma revolução, pois substituem a maior parte da lógica de negócios por novos modelos, introduzindo novos paradigmas econômicos que podem mudar nossa sociedade. Por exemplo, um DAO que possa selecionar e investir de forma autônoma em diferentes startups, administrar o desenvolvimento de negócios e depois vender suas participações nelas para outros fundos e redistribuir os lucros aos seus acionistas. Por fim, as aplicações focadas em Aste et al. (2017) foram financeiras, como o bitcoin.

Bartoletti et al. (2018) analisaram uma coleção de 120 projetos de bem social habilitados para blockchain. Focando em aspectos mensuráveis e objetivos, algumas questões foram analisadas: quais recursos da blockchain são mais comumente usados? Os projetos têm sucesso na captação de recursos? Eles estão fazendo escolhas apropriadas na arquitetura da blockchain? Quantos projetos são lançados ao público e quantos são eventualmente abandonados? Após a coleta, os projetos foram categorizados conforme seu setor de impacto social. As categorias com mais menções foram Filantropia, Ambiente e Inclusão Financeira. A Filantropia inclui plataformas de doação de caridade. Ambiente inclui projetos que têm como principal objetivo a melhoria da qualidade do meio ambiente, bem como projetos que visam a otimização do uso e distribuição de energia. A inclusão financeira compreende plataformas de software para remessas e micro-empréstimos.

Diante das mudanças desencadeadas pelo surgimento das criptomoedas, também surge a necessidade de compreender os possíveis desdobramentos na economia e no comportamento da sociedade [Cardoso et al. 2018]. Há a percepção que outras áreas de estudo já notaram, a capacidade de inovação da blockchain, fora da área da Computação. Cardoso et al. (2018) expuseram o potencial de utilização das soluções trazidas pela blockchain para aplicações no campo econômico, social, jurídico e institucional. Também apresentaram como aplicações potenciais da blockchain: criptomoedas, contratos inteligentes, soluções cartorais, crowdfunding, registro de patentes e marcas, levantamento de informações demográficas e eleitorais, economia, governo e justiça.

Chen et al. (2018) apresentaram um survey da aplicação da blockchain em diferentes domínios, tais como: energia, proteção de direitos autorais, cuidados em saúde, seguro, publicidade e criptomoedas. Especificamente para aplicações sociais, os autores 
discutiram sobre empréstimo de dinheiro de forma não tradicional, veículos e smartphones, música e governo. Essas áreas estão aplicando blockchain para soluções de problemas específicos, segurança e facilidade de uso.

Blockchain também pode ajudar pessoas a melhorarem suas vidas em outros contextos [Gatteschi et al. 2018]. Pode ser usada para registrar dados de genoma e tornar esses dados acessíveis aos proprietários, auxiliando pessoas a mudarem seu estilo de vida, caso seja encontrada uma predisposição para uma determinada doença. Pesquisadores poderiam obter acesso a dados de saúde de exames, tratamentos ou atividades pessoais. Em educação, contratos inteligentes poderiam gerenciar doações financeiras, transferências de dinheiro apenas quando alunos forem aprovados no exame final, registrar as ações dos alunos, garantindo transparência nos contextos de mobilidade ou nos processos de procura de emprego e contratação. Na indústria, blockchain pode ser integrada a BIGDATA para criar sistemas reativos preditivos, coletando e armazenando grandes quantidades de informações para serem processadas posteriormente e tornando-as acessíveis combinando o poder da inteligência artificial e contratos inteligentes. Existem vários aplicativos baseados em blockchain em diferentes setores e contextos [Gatteschi et al. 2018], tais como: gerenciamento de dados pessoais, propriedade intelectual, finanças, negociação e apostas, governo, comércio e cadeia de suprimentos, IoT e cuidados de saúde.

Kshetri e Voas (2018) argumentaram que blockchain possui uma proposta de valor muito maior para o mundo em desenvolvimento do que para o mundo desenvolvido. Isto se deve ao fato que a blockchain possui o potencial para compensar a falta de instituições formais eficazes, como regras, leis, regulamentos e sua aplicação. Sendo assim, blockchain afetará positivamente os países em desenvolvimento. Ela pode ajudar a reduzir a fraude e a corrupção e aumentar os títulos de propriedade legal, o que fornece iniciativas empreendedoras aos mais pobres do mundo. Também pode ajudar as transações financeiras a ocorrerem mais rapidamente e garantir que a ajuda seja distribuída com uma menor chance de roubo e fraude.

Manivannan e Academics (2018) explicaram o termo "Engenharia da Paz" como o trabalho de engenharia em direção à sociedade segura, integrando tecnologias inovadoras, prevenindo conflitos e protegendo a paz na sociedade. O crescimento da tecnologia está sendo testemunhado em todo o mundo, além dos limites através da digitalização dos negócios, conectando pessoas e gerando inovações para melhorar vidas. Blockchain pode ser considerado como uma das melhores inovações para proteger os dados no ordem cronológica. Esses dados são autenticados e armazenados nos blocos. As aplicações discutidas em Manivannan and Academics (2018) foram: serviços financeiros, gerenciamento de ativos, assistência médica, seguros, logística, negócios, rastreamento da cadeia de suprimentos e gerenciamento de recursos humanos.

Tang et al. (2019) identificaram que desafios éticos da blockchain são urgentes e extremamente necessários para garantir que a blockchain seja adotada de forma ética. No entanto, as discussões sobre a ética da blockchain são insuficientes, o que deixa um vazio de compreensão teórica até agora. Uma discussão sistemática sobre a ética das aplicações blockchain foi apresentada e os principais desafios sociais levantados por sua tecnologia e aplicações foram mapeados. Há uma visão geral da pesquisa atual sobre a ética das tecnologias e pesquisa geral sobre blockchain para apresentar brevemente a abordagem dos autores. Um modelo conceitual de pesquisa ética em blockchain foi desenvolvido. 
Os autores esperam que esta pesquisa seja um roteiro inicial para o estudo da ética da blockchain, aumentando a conscientização em tempo hábil e estimulando debates mais aprofundados sobre a ética da blockchain na comunidade de Sistemas de Informação.

\section{Procedimentos Metodológicos}

A estratégia de pesquisa deste trabalho consiste na execução das seguintes sete etapas: (i) Definição de perguntas de partida - identificação de uma visão geral inicial da sociedade, blockchain e aplicações; (ii) Análise preliminar da literatura - análise de trabalhos relacionados que discutiram aplicações da blockchain na sociedade; (iii) Questionário online - coleta de dados demográficos e das questões de partida por parte de pesquisadores em blockchain; (iv) Consolidação dos dados - organização e interpretação inicial dos dados coletados; (v) Análise das questões abertas com literatura - relacionamento dos resultados obtidos com os trabalhos da literatura; (vi) Análise qualitativa - utilização de recursos da Grounded Theory para uma análise qualitativa dos dados coletados; e (vi) Obtenção dos direcionamentos futuros - definição de tendências e perspectivas de pesquisa.

\subsection{Questões de Pesquisa}

Uma das intenções desta pesquisa é traçar um panorama da utilização da blockchain pela sociedade e analisar seus impactos. Para isso, algumas questões de pesquisa foram projetadas, descritas a seguir:

- Onde blockchain pode ser aplicada? - Esta questão tem objetivo de identificar áreas de atuação onde blockchain pode ser utilizada. Por exemplo, a área jurídica pode se beneficiar muito da blockchain para a autenticação e validação de processos. Assim, após a identificação de áreas de atuação e de possíveis aplicações, desenvolveremos um mapeamento das relações e interseções entre áreas. Também poderemos visualizar o alcance da blockchain na sociedade.

- Como blockchain pode melhorar a vida das pessoas? - Algumas áreas atualmente são extremamente dependentes de aplicações ou sistemas. Blockchain tem o poder de melhorar certas características, o que possibilita melhorar ainda mais a vida das pessoas. Nesse contexto, essa questão visa identificar potenciais melhorias seja em aplicações, seja em oportunidades de seu uso, de forma que atividades hoje executadas por software ou processos possam ser melhoradas incrementando a qualidade de vida das pessoas.

- Qual o impacto da utilização da blockchain sobre aplicações tradicionais? - Uma questão que sempre discutida é se vale a pena ou não utilizar blockchain. Ou quando. Ou por que utilizar blockchain se já existem soluções de banco de dados e sistemas distribuídos tradicionais que resolvem os problemas. $\mathrm{O}$ aspecto de custo / benefício da utilização da blockchain é o objetivo desta questão, ou seja, qual seu impacto quando comparada às soluções tradicionais.

\subsection{Questionário}

Inicialmente um questionário online foi projetado para a obtenção de dados, apresentado na Tabela 1, composto por dois grupos de questões: questões demográficas, para identificar o perfil de quem responde à pesquisa; e questões abertas para a opinião. QD1 possuiu como opções nada, pouco, razoável e alto. QD2 possuiu apenas sim e não. As demais questões foram de texto livre, focando nas questões de pesquisa e para opinião. Os dados obtidos foram analisados de maneira quantitativa e qualitativa. 
Tabela 1. Questões demográficas (QD) e questões abertas (QA) do questionário

\begin{tabular}{|l|l|}
\hline QD1 & Qual seu nível de conhecimento dos impactos da utilização da blockchain na sociedade? \\
\hline QD2 & Você acha relevante pesquisas relacionadas a blockchain e sociedade? \\
\hline QA1 & Onde blockchain pode ser aplicada? \\
\hline QA2 & Como blockchain pode melhorar a vida das pessoas? \\
\hline QA3 & Qual o impacto da utilização da blockchain sobre aplicações tradicionais? \\
\hline
\end{tabular}

\subsection{Grounded Theory}

A análise qualitativa deste trabalho foi inspirada nos procedimentos de análise apresentados em Ferreira et al. (2018). O Grounded Theory tem como objetivo criar uma teoria a partir dos dados coletados e analisados sistematicamente, sendo composto por três fases: (1) codificação aberta, (2) codificação axial e (3) codificação seletiva. Na codificação aberta é realizada a quebra, análise, comparação, conceituação e categorização dos dados [Corbin and Strauss 2014]. Nas primeiras fases dessa codificação, uma leitura meticulosa das respostas coletadas é realizada. Cada trecho relevante recebe uma frase ou palavra, formando-se códigos e categorias. Na codificação axial relacionam-se as categorias às suas subcategorias, formando categorias densas, desenvolvidas e relacionadas. E a fase de codificação seletiva origina a categoria ou ideia central do estudo, ou seja, a categoria central da teoria na qual todas estão relacionadas.

O processo de codificação finaliza quando nenhum novo dado adiciona novos conhecimentos na análise das categorias. Apesar da proposta do Grounded Theory ser da construção de teorias, Strauss e Corbin explicam que o pesquisador pode utilizar apenas alguns passos para atingir seu objetivo de pesquisa [Corbin and Strauss 2014]. Por exemplo, quando pesquisadores precisam compreender determinado fenômeno ou situação. Nesta pesquisa, apenas as fases 1 e 2 da codificação serão utilizadas para analisar os dados, visando identificar aspectos e relações em destaque. Este tipo de análise envolve criatividade, experiência e viés do pesquisador. Devido a isto, buscou-se fazer verificações das análises realizadas, sendo estas conduzidas por outro pesquisador.

\section{Resultados}

Para uma visão dos impactos do uso da blockchain, um questionário online foi aplicado com pessoas que pesquisam blockchain (Tabela 1). A pesquisa teve 11 respondentes, todos pesquisadores em blockchain em algum nível (graduação, mestrado ou doutorado em andamento, professor e pesquisador). $\mathrm{O}$ objetivo desse questionário foi verificar a viabilidade da pesquisa e se eles acham interessante saber das relações da blockchain e sociedade. Apesar de poucas respostas, as questões abertas possibilitaram ótimos feedbacks, que colaborarão muito para o prosseguimento da pesquisa. Algumas citações dos participantes foram capturadas para destacar aspectos das questões abertas, sendo que os participantes estão identificados de P1 a P11 para preservar o anonimato.

A Figura 1 exibe uma nuvem de palavras construída com todas as respostas do questionário, apenas com palavras com pelo menos 2 citações. Os comentários a seguir destacam a quantidade de citações de algumas palavras. Excetuando-se a palavra blockchain, percebe-se a presença de dados (8) e informações (4), relacionados ao conteúdo a ser armazenado na blockchain, e segurança (7) como uma das características fortes da blockchain agregando valor. As citações de negócios (3) e processos (3) refle- 


\section{blockchain dados segurança exemplo saúde aplicações informações serviços auditabilidade negócios processos sistemas transações áreas características cartório consenso custos descentralização diversas educação eliminar garantia imutabilidade informação interação logística meio rapidez registro tecnologia tradicionais transparência}

\section{Figura 1. Nuvem de palavras com os destaques das respostas}

tem uma visão que a blockchain já está sendo conhecida ou pensada como uma solução para melhoria das aplicações (5) e serviços (4). Auditabilidade (3), imutabilidade (2), descentralização (2) e transparência (2) são características da blockchain que foram mencionadas, o que já pode ser um indício que as pessoas já estão entendendo seus benefícios. O grande destaque para áreas foi saúde (6), mas houve menções à educação (2). São potenciais áreas de aplicação da blockchain. E por fim, aplicações diversas foram citadas.

Em relação a QD1, 7 pessoas responderam que possuíam um nível de conhecimento dos impactos da utilização da blockchain na sociedade razoável, 3 responderam que o nível de conhecimento era pouco, e apenas 1 respondeu que o nível era alto. Para a QD2, todos responderam que sim, que pesquisas relacionadas com blockchain e sociedade são relevantes. Isso leva a crer que a pesquisa tem importância para ser aprofundada.

A questão QA1 (Onde blockchain pode ser aplicada?) indicou que diversas áreas podem se beneficiar da blockchain. Em relação às áreas específicas que foram citadas, por ordem decrescente de frequência, foram: Saúde (7), Finanças (4), Transportes (4), Educação (2), Logística (2), Cartórios (1), Governo (1), Indústria (1), Judiciário (1), Mercado (1) e Negócios (1). As áreas que mais receberam menções foram saúde, finanças e transportes. Um exemplo de comentário que refletiu bem essa questão foi o de P1: "Em todas as áreas! Finanças, educação, saúde, logística são apenas alguns exemplos". O destaque de finanças é para as criptomoedas, como bitcoin. Entretanto, saúde foi a área mais ressaltada, com registros eletrônicos de dados de saúde. P2 reforçou o lado sociedade com o seguinte discurso: "Em quase todos os âmbitos do meio social, quando se utiliza o conceito de tornar um processo seguro por meio de registro definitivo, pode-se usar blockchain. Temos exemplo de uso na saúde, negócios, veiculos, entre outros".

Na questão QA2 (Como blockchain pode melhorar a vida das pessoas?), diversos benefícios da utilização da blockchain foram citados: Segurança (4), Imutabilidade (3), Eliminação de elementos terceiros (2), Rapidez (2), Redução de custos (2), Auditabilidade (1), Automação (1), Confiança (1), Controle de acesso (1), Descentralização (1), Disponibilidade (1), Integridade (1) e Transparência (1). Muitas dessas melhorias estão relacionadas às características básicas da blockchain. Muito foco para a segurança da informação, como o comentário de P3: "Provendo segurança, controle de acesso e disponibilidade de informações". Algumas respostas foram mais técnicas, mas reforçaram características fundamentais da blockchain, como a resposta de P2: "Blockchain pode fornecer garantias de integridade de uma informação, que muitas vezes pode ser crucial. Por exemplo transações bancárias usando blockchain vão garantir que o registro da transação está imutável, concreto, assim pagador e recebedor tem essas garantias". Um destaque foi para citações para rapidez. Essa rapidez está relacionada à "desburocratização" de pro- 
cessos, muitas vezes pela eliminação de terceiros. P10 destacou esse ponto: "Importância ampla em indústrias, ajudando as empresas a trabalhar com mais inteligência e rapidez". Por fim, alguns discursos foram na direção da redução de custos, com o de P11: "Eliminar ou reduzir custos, como o exemplo dos serviços de cartório. Ajudar na transparência de dados e sistemas etc".

Por fim, a questão QA3 (Qual o impacto da utilização da blockchain sobre aplicações tradicionais?) destacou a melhoria que as aplicações tradicionais teriam com a agregação das características da blockchain. Os itens citados foram: Auditabilidade (3), Segurança (3), Imutabilidade (2), Adaptação (1), Confiança (1), Consenso (1), Descentralização (1), Desempenho (1), Dinamismo (1), Economia (1), Integrar (1), Interação (1), Irrefutabilidade (1), Melhoramento de processos (1), Aumento da qualidade (1), Surgimento de novos negócios (1) e Transparência (1). P1 ressaltou que as aplicações tradicionais sempre existirão e que a qualidade pode aumentar com blockchain: "Elas não vão deixar de existir nunca, mas com a agregação de recursos da blockchain quando possível, a qualidade vai aumentar. Também tem que se considerar o desempenho de aplicações blockchain e quando ela pode ser utilizada". Os processos relacionados às aplicações também foram comentados, como descrito por P2: "Melhoramento de processos. A blockchain traz uma maneira mais concreta e assertiva para realização dos mesmos processos. Um bom exemplo disso são os contratos inteligentes". E a adaptação, apesar de ter sido citada apenas por P3, é algo que terá que ocorrer na migração ou manutenção de aplicações tradicionais para blockchain: "Será necessário adaptação das aplicações tradicionais para incorporar ou integrar soluções baseadas em blockchain para ter os benefícios dessa nova tecnologia". Auditabilidade e segurança foram os mais citados, e são características da blockchain bastante conhecidas e consideradas na academia e indústria, e reforçado pela resposta de P11: "Uma gestão de dados mais confiável, com distribuição replicada totalmente e a possibilidade de auditabilidade. Surge como um tecnologia disruptiva que fornece uma série de características que podem ser essenciais: consenso, auditabilidade, irrefutabilidade etc".

\section{Discussão e Análise}

Nesta seção, os trabalhos relacionados serão comparados com os resultados obtidos na pesquisa, a análise qualitativa será apresentada e discutida, e os direcionamentos da pesquisa serão apresentados.

\subsection{Discussão dos Resultados com Literatura}

A pesquisa, conforme questionário, resultou nas seguintes áreas de aplicação para blockchain: saúde, finanças, transportes, educação, logística, cartórios, governo, indústria, judiciário, mercado e negócios. A Tabela 2 apresenta as áreas apontadas pelos trabalhos relacionados que coincidiram com as áreas citadas no questionário. Nela não estão citadas todas as áreas ou aplicações que os trabalhos relacionados relataram.

Logística foi explicitamente citada em um trabalho. Entretanto, a cadeia de suprimentos em si é uma aplicação que tem se beneficiado muito da blockchain, e foi citada em alguns trabalhos relacionados. A área de saúde foi citada por mais da metade dos trabalhos. Muitas aplicações de e-health tem utilizado recursos da blockchain. Além disso, esta área produz muita pesquisa, então é natural que blockchain esteja sendo utilizada. Finanças esteve presente em todos os trabalhos relacionados, o que era esperado. 
Tabela 2. Comparativo de trabalhos relacionados

\begin{tabular}{|l|l|}
\hline Trabalho Relacionado & Aplicações ou Áreas \\
\hline [Aste et al. 2017] & finanças \\
\hline [Bartoletti et al. 2018] & educação, finanças, saúde \\
\hline [Cardoso et al. 2018] & cartórios, finanças, governo, judiciário \\
\hline [Chen et al. 2018] & finanças, governo, saúde \\
\hline [Gatteschi et al. 2018] & educação, finanças, governo, indústria, saúde \\
\hline [Kshetri and Voas 2018] & finanças, judiciário, negócios \\
\hline [Manivannan and Academics 2018] & finanças, logística, negócios, saúde \\
\hline [Tang et al. 2019] & finanças, mercado, negócios, saúde \\
\hline
\end{tabular}

Isto se deve muito às criptomoedas, aplicação inicial da blockchain, e ao bitcoin. Governo, judiciário, educação e cartórios tiveram menções, muito devido às possibilidades de aplicações e serviços que podem ser melhorados com blockchain. Por fim, indústria, mercado e negócios são áreas que podem ser beneficiar da blockchain em seus processos, gerando uma melhoria dos serviços para o cidadão.

Ao considerar que os dados do questionário refletiram algumas áreas de atuação coincidentes com trabalhos da literatura, podemos considerar que há um alinhamento de ideias. Como a área blockchain é relativamente recente, e os trabalhos relacionados também, então o momento para investir em pesquisa nessa área é favorável.

Apesar do questionário não ter em nenhum momento mencionado ética, nota-se que a sociedade começa a perceber uma necessidade sobre esse assunto, já que a blockchain pode ser utilizada para o compartilhamento de dados pessoais, e a quem esses dados pertencem. Essa discussão tem impacto direto em todas as áreas que utilizam blockchain.

\subsection{Análise Qualitativa}

Para obter um melhor e mais profundo entendimento sobre os resultados das questões abertas, executou-se alguns procedimentos do Grounded Theory. A ideia foi identificar códigos (categorias) e relações. Durante a análise qualitativa, 6 categorias foram identificadas, com a seguinte frequência de citações em ordem decrescente: conceito (20), áreas (11), benefício (8), opinião (7), ação (6), e impacto (5). A Tabela 3 exibe a descrição de cada categoria. As relações identificadas foram apenas três: conceito está relacionado com áreas, conceito é causa de impacto, e conceito é causa de benefícios. Essas relações foram as únicas que puderam ser reforçadas pelas respostas. A Figura 2 apresenta o resultado da análise qualitativa.

Para a relação conceito está relacionado com áreas identificou-se que em vários

Tabela 3. Descrição das categorias identificadas na análise qualitativa

\begin{tabular}{|l|l|}
\hline Categoria & Descrição \\
\hline Ação & são atitudes que o respondente descreveu pelo uso da blockchain \\
\hline Áreas & são aplicações ou domínios onde blockchain pode ser utilizada \\
\hline Benefício & $\begin{array}{l}\text { qualquer vantagem da blockchain para as aplicações e para a sociedade onde quem res- } \\
\text { pondeu não entrou muito no mérito técnico }\end{array}$ \\
\hline Conceito & características técnicas ou estruturais da blockchain, conhecimento teórico ou prático \\
\hline Impacto & é uma consequência do uso da blockchain \\
\hline Opinião & algo mais pessoal que o respondente escreveu, como o sentimento pelo uso da blockchain \\
\hline
\end{tabular}




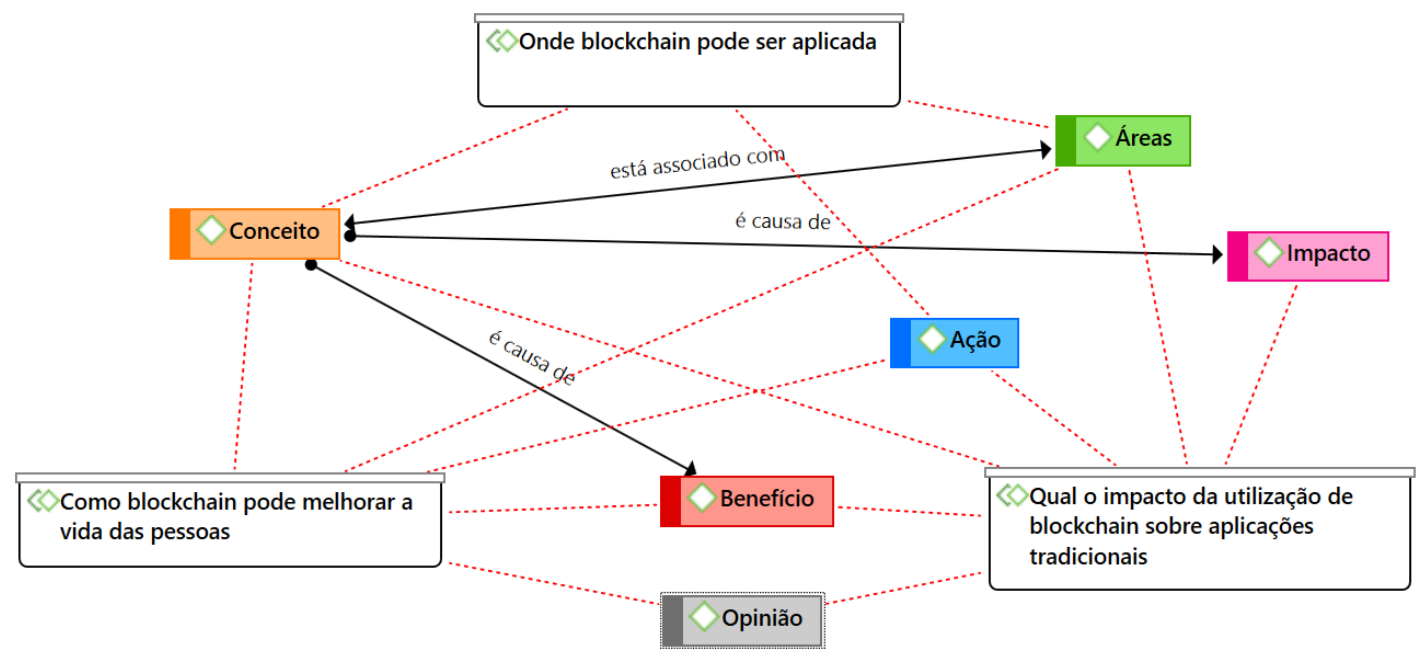

Figura 2. Relações e códigos da análise qualitativa com grounded theory

comentários que todas vez que uma área era citada, geralmente havia alguma característica relacionada. Os seguintes textos de $\mathbf{P 1 1}$ e $\mathbf{P 4}$ respectivamente corroboram com essa relação: "Poderia substituir alguns serviços de cartório, consenso distribuído para disseminação de informação, auditabilidade de dados/sistemas, aplicações de laudos médicos, serviços de localização de objetos (transportadoras) etc" e "A blockchain acaba gerando mais transparência e segurança para os usuários...no caso de um contrato inteligente...oferece agilidade e um histórico completo de um imóvel, por exemplo, informações do tipo quantos donos já tiveram antes".

A relação conceito é causa de impacto demonstrou que alguns respondentes possuíam conhecimento de conceitos da blockchain e conseguiam listar impactos. $\mathrm{O}$ comentário de $\mathbf{P 7}$ reforçou essa relação: "A blockchain pode impactar positivamente em diversas aplicações tradicionais que necessitam de suas características de imutabilidade, descentralização e segurança".

Por fim, a relação conceito é causa de benefícios vai na mesma linha de raciocínio da relação anterior. Em geral, pelas respostas, quem possui conhecimentos em blockchain também soube descrever benefícios de seu uso. P6 redigiu o seguinte comentário sobre essa relação: "Em eliminar os intermediários para baixar custos em diversos setores".

\subsection{Direcionamentos Futuros da Pesquisa e Perspectivas}

Diante dos dados coletados, da revisão da literatura e da análise qualitativa, alguns direcionamentos futuros da pesquisa podem ser tomados para atestar a viabilidade da pesquisa e seu prosseguimento de maneira mais formal.

Em relação a áreas de aplicação, saúde e finanças foram as que se destacaram. Motivado pelo bitcoin, e outras criptomoedas, a área relacionada a finanças, negociações e transações financeiras tende a se expandir bastante. Estudar aspectos humanos e tecnológicos dessas áreas pode ser um boa oportunidade de pesquisa. Na área da saúde, diversas arquiteturas com blockchain estão sendo propostas, por exemplo, Moreira Neto et al. (2020) apresentaram uma arquitetura de e-health com IoT com a agregação de recursos da blockchain e desenvolvimento de aplicações mobile e web. As perspectivas de 
expansão da pesquisa são boas e a integração das áreas tende a crescer.

Todas as relações identificadas pela análise qualitativa possuem a categoria "conceito" envolvida. Assim, estudar conceitos da blockchain, suas características, como elas podem ser aplicadas nos sistemas atuais, como elas impactam no desenvolvimento, como elas melhoram a qualidade das aplicações e consequentemente dos usuários são possíveis itens a serem investigados. Porém, muitas vezes eles atingem a sociedade de maneira transparente, indiretamente por meio das aplicações e serviços.

Avaliar a viabilidade de se utilizar blockchain é um aspecto importante, pois há um custo computacional grande. Alguns trabalhos na literatura discutiram sobre esta relação, como [Pedersen et al. 2019], com critérios para se decidir se usar blockchain vale a pena ou não. Esse tipo de validação é necessária para que as aplicações não sejam prejudicadas.

A blockchain aos poucos está tomando um rumo para adentrar em todas as áreas do conhecimento, sejam exatas, saúde, sociais e humanas. As possibilidades de aplicação estão se ampliando, o que leva a um contexto interdisciplinar entre áreas, onde há um objetivo comum e demais áreas colaboram para seu atendimento.

O alcance da blockchain atinge a diversos aspectos, sejam culturais ou históricos. Um exemplo é a utilização da blockchain para a proteção à propriedade intelectual de obras de arte. Do ponto de vista econômico já é comum aplicações financeiras, muito devido à fama do bitcoin. Juridicamente também a blockchain está ganhando espaço, por exemplo com a autenticação de documentos digitais e transações de contratos em cartórios virtuais.

Em relação à ética, Tang et al. (2019) reforçaram que uma discussão sobre a conscientização e compreensão das questões éticas da blockchain entre acadêmicos e profissionais é necessária. Muito disso está relacionado ao compartilhamento de dados dos usuários, como aplicações médicas que compartilham resultados de exames com pacientes e médicos. A privacidade de dados é um aspecto importante a ser considerado, e uma discussão sobre de quem é a posse dos dados, e como eles podem ser utilizados.

\section{Conclusão}

Esta pesquisa é um ensaio inicial sobre blockchain e seus impactos na sociedade. Observou-se que atualmente a tecnologia está ampliando sua utilização em diversas áreas, e que a sociedade está percebendo seus benefícios. Mesmo com poucas respostas no questionário, os resultados são um indício que a pesquisa deve prosseguir e ser aprofundada. Percebeu-se que saúde e finanças foram as áreas mais mencionadas e que há uma necessidade de discutir sobre blockchain e seu uso na sociedade.

Como limitações da pesquisa, este trabalho contou com poucas respostas no questionário, sendo necessário mais respostas para melhores conclusões e direcionamentos. Adicionalmente, as respostas foram de pesquisadores em blockchain, que representam apenas uma parcela da sociedade. Além disso, a maioria das respostas foi de apenas uma região geográfica, o que pode caracterizar apenas um grupo, tendo que a pesquisa ser ampliada para mais regiões. Por fim, a pesquisa na literatura foi realizada apenas com uma consulta simples a bases digitais, sendo necessário um maior refinamento para a obtenção de mais trabalhos, dados mais aderentes à pesquisa, e adição de mais bases.

Temos consciência que estes resultados são preliminares, mais para sondar o inte- 
resse pela pesquisa, e que não teve participação de pessoas que não pesquisam em blockchain. A aplicação de um questionário mais elaborado com diversas outras pessoas, grupos de pesquisa, academia e indústria é o próximo passo, e assim atingir o máximo de respondentes das mais diversas áreas. Como trabalhos futuros pretende-se ampliar o estudo, analisando o impacto social por meio de questionários sobre a tecnologia e sobre sua relevância em áreas diversas, investindo nos direcionamentos futuros apresentados.

\section{Referências}

Aste, T., Tasca, P., and Di Matteo, T. (2017). Blockchain technologies: The foreseeable impact on society and industry. Computer, 50(9):18-28.

Bartoletti, M., Cimoli, T., Pompianu, L., and Serusi, S. (2018). Blockchain for social good: A quantitative analysis. In Proceedings of the 4th EAI International Conference on Smart Objects and Technologies for Social Good, Goodtechs '18, page 37-42.

Cardoso, A. A., Costa, E. S., and Silveira, F. A. (2018). Criptomoedas e blockchain no processos de inovação social. In II Congresso Sul Catarinense de Administração e Comércio Exterior.

Chen, W., Xu, Z., Shi, S., Zhao, Y., and Zhao, J. (2018). A survey of blockchain applications in different domains. In Proceedings of the 2018 International Conference on Blockchain Technology and Application, pages 17-21.

Corbin, J. and Strauss, A. (2014). Basics of Qualitative Research: Techniques and Procedures for Developing Grounded Theory. SAGE Publications, 4 edition.

Ferreira, T., Viana, D., Fernandes, J., and Santos, R. (2018). Identifying emerging topics and difficulties in software engineering education in brazil. In Proceedings of the XXXII Brazilian Symposium on Software Engineering, SBES '18.

Gatteschi, V., Lamberti, F., Demartini, C., Pranteda, C., and Santamaría, V. (2018). To blockchain or not to blockchain: That is the question. IT Professional, 20(2):62-74.

Kshetri, N. and Voas, J. (2018). Blockchain in developing countries. IT Professional, 20(2):11-14.

Manivannan, K. and Academics, D. (2018). Contribution of blockchain technology to the growth of the society. In 2018 World Engineering Education Forum - Global Engineering Deans Council (WEEF-GEDC), pages 1-3.

Moreira Neto, M., Coutinho, E. F., Moreira, L. O., and de Souza, J. N. (2020). Toward blockchain technology in iot applications: An analysis for e-health applications. In Internet of Things. A Confluence of Many Disciplines, pages 36-50.

Pedersen, A. B., Risius, M., and Beck, R. (2019). A ten-step decision path to determine when to use blockchain technologies. MIS Quarterly Executive, 18(2).

Tang, Y., Xiong, J., Becerril-Arreola, R., and Iyer, L. (2019). Blockchain ethics research: A conceptual model. In Proceedings of the 2019 on Computers and People Research Conference, SIGMIS-CPR '19, page 43-49.

Xie, J., Tang, H., Huang, T., Yu, F. R., Xie, R., Liu, J., and Liu, Y. (2019). A survey of blockchain technology applied to smart cities: Research issues and challenges. IEEE Communications Surveys \& Tutorials, 21(3):2794-2830. 